\title{
3 Research Square

\section{Enhanced Anti-Cancer Potency Using A Combination of Oleanolic Acid and Maslinic Acid to Control Treatment Resistance in Breast Cancer.}

Cıgır Biray Avci ( $\nabla$ cigir.biray@ege.edu.tr)

Ege University Faculty of Medicine: Ege Universitesi Tip Fakultesi https://orcid.org/0000-0003-32192425

\section{Fatma Sogutlu}

Ege University Faculty of Medicine: Ege Universitesi Tip Fakultesi

\section{Neslihan Pinar Ozates}

Ege University Faculty of Medicine: Ege Universitesi Tip Fakultesi

\section{Behrouz Shademan}

Ege University Faculty of Medicine: Ege Universitesi Tip Fakultesi

Cumhur Gunduz

Ege University Faculty of Medicine: Ege Universitesi Tip Fakultesi

\section{Research Article}

Keywords: Breast cancer, mTOR signal pathway, Oleanolic acid, Maslinic acid.

Posted Date: November 23rd, 2021

DOI: https://doi.org/10.21203/rs.3.rs-1077510/v1

License: (c) (i) This work is licensed under a Creative Commons Attribution 4.0 International License. Read Full License 


\section{Abstract}

The phosphatidylinositol 3-kinase/AKT/mammalian target of Rapamycin (PI3K/AKT/mTOR) pathway is a complex intracellular metabolic pathway leading to cell growth, and tumor proliferation plays an essential role in drug resistance in breast cancer. Therefore, in the present study, the anti-cancer effects of oleanolic acid (OA) and maslinic acid (MA) were investigated to improve the performance of the treatment strategy. We investigated the effect of OA and MA on cell viability using the WST-1 method. The synergistic effect of the combination was analyzed by isobologram analysis. In addition, the effects of the two compounds, individually and in combination, on apoptosis, autophagy, and the cell cycle were investigated in MCF7 cells. In addition, changes in the expression of PI3K/AKT/mTOR genes involved in apoptosis, cell cycle and metabolism were determined by quantitative RT-PCR. MA, OA, and a combination of both caused G0/G1 arrest. Apoptosis also increased in all treated groups. The autophagosomal LC3-II formation was induced 1.74-fold in the MA-treated group and 3.25-fold in the MAOA-treated group. The combination treatment resulted in increased expression of genes such as GSK3B, PTEN, CDKN1B and FOXO3 and decreased expression of IGF1, PRKCB and AKT3 genes. The results showed that the combination of these two substances showed the highest synergistic effect at the lowest dose and using MA-OA caused cancer cells to undergo apoptosis. The use of combination drugs may reduce the resistance of cancer cells to treatment. However, further studies are needed to clarify cases.

\section{Introduction}

Breast cancer is one of the most common cancers that cause death in women [1]. Breast cancer is classified into molecular subclasses based on hormone receptor status and epidermal growth factor 2 (HER2) status. Lumen A (ER+, HER2-) breast cancer is one of the most common types [2]. The $\mathrm{PI} 3 \mathrm{~K} / \mathrm{Akt} / \mathrm{mTOR}$ pathway is essential in mammalian cells that control growth through growth stimuli. Factors such as increased growth factors, mutations, loss of gene function, or activation of kinase genes cause inhibition of the pathway leading to carcinogenesis [3, 4]. The PI3K/Akt/mTOR pathway has been identified as a cancer resistance pathway $[5,6]$. In patients receiving hormone therapy for breast cancer, the PI3K/Akt/mTOR pathway has been identified as an escape pathway that causes tumor cells to become resistant to treatment [7]. Therefore, the PI3K/Akt/mTOR pathway can be a target for chemotherapy. However, the potential of natural products as a specific drug is different and using natural substances in combination has a significant effect and minimizes the toxicity and side effects of chemotherapeutic drugs $[8,9]$.

The OA and MA, found in over 1600 plant species, have been included in the pentacyclic triterpenes of the Olean type group. Olea europaea $L$ is the primary source of both natural compounds. Besides biological activities such as antiviral, anti-inflammatory, antidiabetic, antimicrobial, and cardioprotective properties with a proven pharmacological safety profile, OA (31-44\%) and MA (55-68\%), which have the highest density among the six pentacyclic triterpenes found mainly in the intracuticular part of the olive, have anti-cancer properties $[10,11]$. 
In the studies conducted, the efficacy of $\mathrm{OA}$ on malignant gliomas and hematological malignancies in breast, hepatocellular, lung, colon, pancreatic, and gallbladder cancers were demonstrated and found to affect cell survival via PI3K/Akt/mTOR, NF-KB, TGF- $\beta$, ERK/JNK/p38 MAPK, signaling pathways [12, 13]. The anti-cancer effects of MA on melanoma, colon, ovarian, lung, oral carcinoma, liver, and breast cancers were investigated and found to have antiproliferative, cytostatic, apoptotic, and autophagic effects via JAK/STAT3, AMPK/mTOR, p38 MAPK, and NF-KB signaling pathways [14-16]. This study aimed to determine the effects of OA and MA on toxicity, apoptosis, and autophagy in the MCF7 cell line, both individually and in combination. In addition, changes in the expression of PI3K / AKT / mTOR genes involved in apoptosis, cell cycle, and metabolism were determined by quantitative RT-PCR.

\section{Materials And Methods}

\section{Cell line and culture conditions}

The MCF7 cell line was purchased from the American Type Culture Collection (ATCC\# HTB-22 ${ }^{\text {TM }}$ ). Cells were cultured in DMEM (Cat. No. 01-052-1A, Biological Industries, USA) containing 2 mM L-glutamine, 1\% penicillin-streptomycin and $10 \% \mathrm{FBS}$ in an incubator with $95 \%$ humidity, $37^{\circ} \mathrm{C}$ and $5 \% \mathrm{CO}_{2}$. The cultured cells were examined with an "inverted" microscope. OA (cat. no.: 05504) and MAs (cat. no.: M6699) were purchased from Sigma-Aldrich. OA and MA were soluble in DMSO at $44 \mathrm{mM}$ and $10 \mathrm{mM}$, respectively, and were stored diluted at $-20^{\circ} \mathrm{C}$. The subsequent dilutions were prepared in DMEM.

\section{Cell viability assay}

Cells were seeded in a 96-well plate $(6.2 \times 103$ cells per well $)$ and incubated for one day under standard conditions. MCF7 cancer cells were treated with different concentrations of OA (462-184.8 $\mu \mathrm{M})$ and MA $(100-40 \mu \mathrm{M})$ for 48 and 72 hours. After this period, the cytotoxic effect of OA, MA, and their combination on the MCF7 cell line was quantified using the cell proliferation reagent WST-1 (Cat. No.: 11644807001, Roche). $10 \mu \mathrm{L} /$ well of WST-1 solution was added and incubated for 1 hour. Optical density (OD) was measured every 15 minutes after incubation using a microplate reader (Multiskan FC, Thermo) in the reference range of $450 \mathrm{~nm}$ absorbance $620 \mathrm{~nm}$. IC50 doses that reduced cell proliferation by $50 \%$ were determined for OA and MA in the MCF7 cell line.

\section{Isobologram}

The interactions between $\mathrm{OA}$ and MA were evaluated using the isobologram analysis method, which was used to determine the combination's additive, synergistic, and antagonistic effects. The combined mixture of MA and OA was 100:462, respectively.

\section{Cell cycle assay}

The effect of $\mathrm{OA}$ and MA on different stages of the cell cycle was determined by flow cytometry. Briefly, cells $(3.5 \times 105)$ were seeded into a 6-well plate and allowed to adhere for 24 hours. Then, 72 hours after treatment with $\mathrm{OA}, \mathrm{MA}$, and MA-OA, cells were removed with trypsin and centrifuged at $400 \times \mathrm{g}$ for 5 
minutes. The cells were rinsed twice with $1 \times$ PBS. According to the protocol of the kit (cat. no. 340242, BD Biosciences), cells were washed twice with cell cycle buffer. Solutions A, B, and C were added to each. The cells were analyzed by flow cytometry (Becton-Dickinson, USA). The group not treated with natural substances served as controls.

\section{Apoptosis assay}

Evaluation of the apoptotic effect of OA and MA on MCF7 cell lines through the phosphatidylserine (PS) in the cell membrane was performed according to the manual of the FITC Annexin $V$ Apoptosis Detection Kit (Cat. No.: 556547, BD Pharmingen, USA) protocol by counting $3.5 \times 105$ cells on the BD ACCURI C6 flow cytometer (BD Biosciences Pharmingen). After OA and MA were added to the cells at the desired dosage, their apoptotic effect was examined for 72 hours. The group that was not treated with OA and MA was considered as the control group.

\section{Autophagy assay}

The Premo Autophagy Tb/GFP TR-FRET LC3B Expression Kit (cat. no. A14070; Invitrogen; USA) was used to determine the effect of OA, MA, and MA-OA on autophagosome formation in MCF7 cells. Cells supplemented with Optimum I with $10 \%$ serum were mixed with different concentrations of LC3B-GFP BacMam and allowed to attach in a 96-well plate. After 24 hours, the IC50 values of the natural compounds and the ED50 values of the combination were applied to the cells of the dose group. In the negative control group, chloroquine was added to the cells to inhibit lysosome activity by accumulating LC3B II. The growth medium was also added to the cells of the control group. Cells were incubated at room temperature for 2 hours after adding lysis buffer to each well of the 96-well plate. TR-FRET emission was measured using the $332 \mathrm{~nm}$ excitation, $518 \mathrm{~nm}$, and $488 \mathrm{~nm}$ emission filters in the Varioskan Flash Multimode Microplate Reader (Thermo Scientific).

\section{Gene expression}

The MCF7 cell line was treated with OA and MA for $72 \mathrm{~h}$ to examine the expression of AKT3, BAD, EIF2AK2, GSK3 3 , FOX03, PRKCB, PTEN, TLR4, CDKN1B genes by quantitative Real-Time PCR (qRT-PCR). In brief, total RNA was extracted from control and treated cells using the RNeasy Plus Mini Kit (Cat. No. 74134, Qiagen, USA) according to the manufacturer's instructions. The quantity and quality of extracted RNA were assessed by nanodrop and electrophoresis on a $1 \%$ agarose gel. The RT2 First Strand Kit (cat. no. 330401; Qiagen, USA) was used for CDNA synthesis. The specific primers are listed in Table 1. The GAPDH gene was considered an endogenous control gene for normalization of the AKT3, BAD, EIF2AK2, GSK3 $\beta$, FOXO3, PRKCB, PTEN, TLR4, and CDKN1B genes. The threshold (Ct) was determined for the genes. The comparative $2^{-\Delta \Delta C t}$ threshold cycle method was used for data analysis. 
Table 1

Primer sequences for the studied genes.

\begin{tabular}{|c|c|c|}
\hline Gene & \multicolumn{2}{|c|}{ Primer sequence } \\
\hline \multirow[t]{2}{*}{ AKT3 } & Forward & 5'-TGGACAAAGATGGCCACATA-3' \\
\hline & Reverse & 5'-ATCAAGAGCCCTGAAAGCAA-3' \\
\hline \multirow[t]{2}{*}{ BAD } & Forward & 5'-ACCCGGCAGACAGATGAG-3' \\
\hline & Reverse & 5'-СТTССТСTCССАCCGTAGC-3' \\
\hline \multirow[t]{2}{*}{ EIF2AK2 } & Forward & 5'-AAAAATCAGGAGACCCTGGCTA-3' \\
\hline & Reverse & 5'-TCTTCCCGTATCCTGGTTGGA-3' \\
\hline \multirow[t]{2}{*}{ F0X03 } & Forward & 5'-ACGGTGTTCGGACCTTCATC-3' \\
\hline & Reverse & 5'-TGCTGGCCTGAGACATCAAG-3' \\
\hline \multirow[t]{2}{*}{ GSK3B } & Forward & 5'-GGAACTCCAACAAGGGAGCA-3' \\
\hline & Reverse & 5'-TTCGGGGTCGGAAGACCTT A-3' \\
\hline \multirow[t]{2}{*}{ PRKCB } & Forward & 5'-GCCTACCCCAAGGTCCATGT-3' \\
\hline & Reverse & 5'-CTTGGTCATGAGCCCTTTG-3' \\
\hline \multirow[t]{2}{*}{ PTEN } & Forward & 5'-ACCAGGACCAGAGGAAACCT-3' \\
\hline & Reverse & 5'-GCTAGCCTCTGGATTTGACG-3' \\
\hline \multirow[t]{2}{*}{ TLR4 } & Forward & 5'-САСTGTTCTTCTCCTGCCTGAC-3' \\
\hline & Reverse & 5'-TGG TTGAAGAAGGAATGTCATC-3' \\
\hline \multirow[t]{2}{*}{ CDKN1B } & Forward & 5'-GGGTAGAGTTGGGGGTAG-3' \\
\hline & Reverse & 5'-ACAAАCСТАСТСТААСТААССТ-3' \\
\hline \multirow[t]{2}{*}{ IGF1 } & Forward & 5'-GGCATAGCTGGCCAAACAA-3' \\
\hline & Reverse & 5'-CACTTGGGAGAAGGCTTAGAATAAA-3' \\
\hline \multirow[t]{2}{*}{ GAPDH } & Forward & 5'-CTGACTTCAACAGCGACACC-3' \\
\hline & Reverse & 5'-TAGCCAAATTCGTTGTCATACC-3' \\
\hline
\end{tabular}

\section{Statistical analysis}

Student t-test was used for statistical analysis of gene expression. A one-way ANOVA was performed for analysis of autophagy results. IC50 values were calculated using CalcuSyn v.2.1 software. The $p<0.05$ was considered statistically significant. 


\section{Results}

\section{Evaluation of Cell viability}

In MCF7 cells, the IC50 values of OA and MA were $70.3 \mu \mathrm{M}$ and $291 \mu \mathrm{M}$, respectively (Fig. 1). The combination indices of the fractions affected at $50 \%, 75 \%$, and $90 \%$ were $0.612,0.842$, and 1.159 , respectively, and the experiments were continued with the ED50 value that showed the highest synergistic effect (Fig. 2).

\section{Evaluation of apoptosis}

When apoptosis was assessed by the annexin $\mathrm{V}$ method, apoptosis was $3.1 \%$ in the untreated group, $25.6 \%$ in the group treated with the IC50 value of MA, and $17.2 \%$ in the group treated with the IC50 value of OA. Apoptosis in the $50 \%$ affected fraction of the two natural compounds was $30.1 \%$. In the breast cancer model, OA, MA, and the combination induced apoptosis by 8.25 -fold, 5.59 -fold, and 9.7-fold, respectively (Fig. 3).

\section{Evaluation of Autophagy}

The effect of IC50 values of the natural compounds and ED50 value of the combination on autophagy was compared with the untreated group. Autophagosomal LC3-II formation was induced 1.74-fold in the group treated with MA, 0.37-fold in the group treated with oleanolic acid, and 3.25-fold in the combination group (Fig. 4).

\section{Evaluation of cell cycle}

When the effect of the natural compounds and the combination on the cell cycle was studied in MCF7 cells, it was found that the cell population was $65.2 \%$ in G0/G1 phase, $14.5 \%$ in the S phase and $20.1 \%$ in the $\mathrm{G} 2 / \mathrm{M}$ phase. The cell population in G0/G1 phase was increased to $72.1 \%$ by MA, $72.5 \%$ by OA, and $73.2 \%$ by the combination treatment. It was found that the natural compounds and the combination caused G0/G1 arrest (Fig. 5).

\section{Evaluation of genes involved in PI3K / AKT / mTOR signaling pathway}

The effect of OA, MA, and their combination on the expression of genes involved in the PI3K / AKT / mTOR signaling pathway in MCF7 cells was investigated. It was found that the expression of BAD, EIF2AK2, FOX03, GSK3 $\beta$, PTEN, CDKN1B, GSK3 $\beta$ genes after MA increased 2.66-fold, 2.94-fold, 5.52-fold, 3.06-fold, 5.98-fold, 1.64-fold, 3.06-fold, and the AKT3, PRKCB, and TLR4 genes decreased 3.89-fold, 4.32fold, 9.04-fold, respectively. The expression of BAD, EIF2AK2, FOXO3, GSK3 $\beta$, PTEN, CDKN1B, GSK3 $\beta$ genes after $O A$ treatment increased 2.61-fold, 2.36-fold, 4.85-fold, 2.70-fold, 5.05-fold, 2.90-fold, 2.70-fold and AKT3, PRKCB, TLR4 genes decreased 4.11-fold, 3.92-fold, 2.36-fold, respectively. In the combinationtreated MCF7 cells, the expression of BAD, EIF2AK2, FOXO3, GSK3 $\beta$, PTEN, CDKN1B, and GSK3 $\beta$ genes was observed to increase 3.01-fold, 2.46-fold, 6.27-fold, 2.49-fold, 4.71-fold, 2.89-fold, 2.49-fold, 
respectively. AKT3, PRKCB, TLR4, IGF1 genes down-regulated 4.88-fold, 4.54-fold, 6.88-fold, 8.96-fold, respectively (Fig. 6).

\section{Discussion}

Natural and synthetic compounds are used as chemotherapeutic agents and are one of the treatment options in cancer treatment $[17,18]$. However, the toxic side effects of synthetic chemotherapeutic agents and the stringent approval procedures before administering them to patients have led researchers to focus on natural compounds to provide safer alternatives for cancer treatment and shorten the drug approval time [19].

The studies conducted have shown the therapeutic potential of many phytochemicals against cancer. The triterpenoids found in rarely consumed fruits, vegetables, and plants, representing the group of phytochemicals, and belonging to the oleanane-type terpenoid group, are easy to find and inexpensive. They also show that their chemo preventive and antitumor activities pave the way for their use as natural anti-cancer agents [20]. Awareness of the important role of the PI3K/Akt/mTOR pathway in breast cancer survival and proliferation and evidence that the oleanane derivatives $\mathrm{OA}$ and MA target the $\mathrm{PI3K} / \mathrm{Akt} / \mathrm{mTOR}$ pathway have been found in attractive breast cancer treatments [16]. Awareness of the important role of the PI3K/Akt/mTOR pathway in breast cancer survival and proliferation and evidence that the oleanane derivatives OA and MA target the PI3K/Akt/mTOR pathway have been found in attractive breast cancer treatments [16]. When OA and MA were used separately and in combination in the MCF7 cell line, it was found that the combination therapy showed a significant synergistic effect. In addition, a dose reduction was observed when the two cases were combined compared with a single treatment. We speculate that the synergistic effect of the combination and the increase in dose reduction index may be related to the distal/proximal targeting of the PI3K / AKT / mTOR pathway with OA and MA.

The PI3K / AKT / mTOR pathway is one of the effective centers for modulating proteins that regulate cell cycle and apoptosis and determine cell fate (Fig. 7). Phosphorylated AKT suppresses apoptosis by playing a role in regulating downstream molecules such as BAD, caspase 9, caspase 3 and FOXO3[21]. The expression of BAD, caspase- 9 and caspase- 3 at the gene and protein levels in MCF7 was examined by targeting the PI3K/AKT pathway with tanshinone, and it was found that the expression of these proapoptotic structures was increased [22]. In the study conducted by Feng et al. investigating the effect of $\mathrm{OA}$ in lung adenocarcinomas, the natural products were found to exhibit apoptotic effect by downregulating $\mathrm{BCl} 2$ and upregulating $\mathrm{Bax}$ and $\mathrm{BAD}$ [23]. The upregulation of $\mathrm{FOXO3}$, which is considered a benchmark for inhibition of the PI3K/AKT pathway and activation of AMPK, is also one of the transcription factors that regulate apoptosis by translocating from the cytoplasm to the nucleus in response to AKT [24]. When the synthetic derivatives of OA and MA were used by Deeb et al. to target prostate cancer, FOXO3, the downstream target of AKT, mTOR and NF-KB, was inhibited [25]. In our study, the expression of transcription factor $\mathrm{FOXO} 3$ increased with different treatments and the combination of $\mathrm{OA}$ and MA. However, some observed that their combination with each other had a synergistic effect on 
FOXO3 expression. A significant increase in apoptosis of MCF7 cells was observed after combination therapy with $O A$ and MA. All this evidence suggests that the combination of the two compounds can increase the expression of FOXO3. In addition, acting on the PI3K / AKT / mTOR pathway increases apoptosis in MCF7 cells.

AKT, one of the leading centers of the PI3K pathway, stimulates the repression of p21 and p27, which act via FOXO3 in cell cycle progression, and increases the transcription of cyclin D by inactivating GSK3 through its phosphorylation and facilitates the transition of the cell cycle from $\mathrm{G} 1$ to S phase [26]. Kim et al. have claimed that breast cancer cells treated with oleanolic acid arrest in the $\mathrm{G} 1$ phase of the cell cycle due to activation of p21 and p27 and inhibition of p-AKT and cyclin D1 [12]. In a study that investigated the effect of OA on prostate cancer in vivo and in vitro, G0/G1 arrest was due to decreased GSK3 $\beta$ activity mediated by AKT and decreased cyclin D expression [13]. We found that G0/G1 arrest occurred upon treatment of natural compounds by the studies in the literature and that the combination slightly increased G0/G1 phase compared to mono-treatment. In MCF7 cells, the enhancement of G0/G1 arrest by treatment with OA, MA and their combinations could result from 1) downregulation of AKT3 gene expression, 2) upregulation of the gene encoding GSK3 $\beta$ protein that blocks cyclin D coding, 3) reduction of FOXO3 expression, 4) downregulation of the gene encoding p27.

Besides autophagy, which is known as a cell death pathway of the ll-type, it can be modulated by various signaling pathways. The main modulator for triggering autophagy is the accepted mTOR, which can be phosphorylated by the PI3K/AKT pathway and various signaling pathways [27]. The mTOR pathway contributes to cell survival by inhibiting the formation of autophagosomes or autolysosomes in the ll-type death pathway. With inhibition of mTOR, this process is reversed [28]. LC3- II is responsible for phagophore expansion in the autophagy process, and cargo recognition by binding to the autophagosome membrane is considered a marker for autophagosome formation [29]. Liu et al. found that the expression of LC3- II was increased by modulating the mTOR and JNK signaling pathway of OA in A549 cells [30]. Farooqi et al. used OA and MA in pancreatic cancer cells and found that OA induced protective autophagy in the cell, while MA induced autophagic cell death [31]. The present study found that OA slightly increased autophagosomal LC3- II formation compared with the untreated group, while it increased MA. The combination contributed to II cell death compared with mono-treatment. Although the only difference between the two natural compounds in terms of chemical structure is a hydroxyl group, they can induce autophagy and apoptosis to different degrees. The coexistence of these two triterpenes and the simultaneous consumption of the same nutriment may show a strong survival inhibitory effect in the luminal A type cancer model. In agreement with the studies performed, we found that PRKCB expression decreased inversely proportional to the increasing autophagy after natural compounds and the combination treatment. PRKCB suppresses autophagy after receiving signals from IGFR1, Nox2 or ERK1/2 [32]. There was no change in IGF1 expression in the current study when OA and MA were administered alone, but we found that IGF1 was significantly down-regulated after combination treatment. The reason for the increased autophagosome structure after two natural compounds in MCF7 cells might be the reduction of the expression of PRKCB in the downstream region of the pathway and IGF1 in the upstream region of the pathway. 


\section{Conclusion}

The role of the PI3K / AKT / mTOR pathway is well known as a trigger for breast cancer, and it is also one of the most important pathways to establish the mechanisms of cancer resistance to treatment. Therefore, blocking various nodes in this pathway may be a suitable and valuable treatment for breast cancer. Using the combination of OA and MA decreased the resistance of cancer cells to treatment and caused cancer cells to go into apoptosis and cell cycle arrest. The combination of these two substances showed the highest synergistic effect at the lowest dose. Our study suggests that the combination of OA and MA may be a promising anti-cancer drug for treating breast cancer patients. However, more studies are needed to understand more facts. We recommend that other breast cancer cell lines be studied.

\section{Declarations}

\section{Author contributions}

CBA and CG Study conception and design; FS, NP, and BS Methodology and Data curation; FS, and NP Writing - original draft; CBA Writing - review \& editing.

\section{Data availability}

The data and materials used in this study are available.

\section{Conflict of interest}

The authors declare no conflict of interest.

\section{References}

1. Azamjah N, Soltan-Zadeh Y, Zayeri F. Global trend of breast cancer mortality rate: a 25-year study. Asian Pacific journal of cancer prevention: APJCP. 2019; 20(7):2015.

2. Yersal O, Barutca S. Biological subtypes of breast cancer: Prognostic and therapeutic implications. World J Clin Oncol. 2014; 5(3):412.

3. Yang J, Nie J, Ma X, Wei Y, Peng Y, Wei X. Targeting PI3K in cancer: mechanisms and advances in clinical trials. Mol Cancer. 2019; 18(1):1-28.

4. Shorning BY, Dass MS, Smalley MJ, Pearson HB. The PI3K-AKT-mTOR pathway and prostate cancer: At the crossroads of AR, MAPK, and WNT signaling. Int J Mol Sci. 2020; 21(12):4507.

5. Shi X, Wang J, Lei Y, Cong C, Tan D, Zhou X. Research progress on the PI3K/AKT signaling pathway in gynecological cancer. Mol Med Rep. 2019; 19(6):4529-35.

6. Ozdemir Kutbay N, Biray Avci C, Sarer Yurekli B, Caliskan Kurt C, Shademan B, Gunduz C, Erdogan M. Effects of metformin and pioglitazone combination on apoptosis and AMPK/mTOR signaling pathway in human anaplastic thyroid cancer cells. J Biochem Mol Toxicol. 2020; 34(10):e22547. 
7. Kanaizumi H, Higashi C, Tanaka Y, Hamada M, Shinzaki W, Azumi T, Hashimoto Y, Inui H, Houjou T, Komoike Y. PI3K/Akt/mTOR signalling pathway activation in patients with ER positive, metachronous, contralateral breast cancer treated with hormone therapy. Oncol Lett. 2019; 17(2):1962-8.

8. Varghese E, Samuel SM, Abotaleb M, Cheema S, Mamtani R, Büsselberg D. The "yin and yang" of natural compounds in anticancer therapy of triple-negative breast cancers. Cancers. 2018; 10(10):346.

9. Ozates NP, Soğutlu F, Lerminoglu F, Demir B, Gunduz C, Shademan B, Avci CB. Effects of rapamycin and AZD3463 combination on apoptosis, autophagy, and cell cycle for resistance control in breast cancer. Life Sci. 2021; 264:118643.

10. Fiad S, El-Hamidi M. Six pentacyclic triterpenes in mature olive fruits" Picual". Am J Food Technol. 2015; 10(1):1-3.

11. Žiberna L, Šamec D, Mocan A, Nabavi SF, Bishayee A, Farooqi AA, Sureda A, Nabavi SM. Oleanolic acid alters multiple cell signaling pathways: implication in cancer prevention and therapy. Int $\mathrm{j}$ mol sci. 2017; 18(3):643.

12. Kim GJ, Jo HJ, Lee KJ, Choi JW, An JH. Oleanolic acid induces p53-dependent apoptosis via the ERK/JNK/AKT pathway in cancer cell lines in prostatic cancer xenografts in mice. Oncotarget. 2018; 9(41):26370.

13. Li X, Song Y, Zhang P, Zhu H, Chen L, Xiao Y, Xing Y. Oleanolic acid inhibits cell survival and proliferation of prostate cancer cells in vitro and in vivo through the PI3K/Akt pathway. Tumour Biol. 2016; 37(6):7599-613.

14. Wang D, Tang S, Zhang Q. Maslinic acid suppresses the growth of human gastric cells by inducing apoptosis via inhibition of the interleukin- 6 mediated Janus kinase/signal transducer and activator of transcription 3 signaling pathway. Oncol let. 2017; 13(6):4875-81.

15. Allouche Y, Warleta F, Campos M, Sanchez-Quesada C, Uceda M, Beltran G, Gaforio JJ. Antioxidant, antiproliferative, and pro-apoptotic capacities of pentacyclic triterpenes found in the skin of olives on MCF-7 human breast cancer cells and their effects on DNA damage. J Agric Food Chem. 2011; 59(1):121-30.

16. Wu J, Yang C, Guo C, Li X, Yang N, Zhao L, Hang H, Liu S, Chu P, Sun Z, Sun B. SZC015, a synthetic oleanolic acid derivative, induces both apoptosis and autophagy in MCF-7 breast cancer cells. Chem Biol Interact. 2016; 244:94-104.

17. Jadid MF, Shademan B, Chavoshi R, Seyyedsani N, Aghaei E, Taheri E, Goleij P, Hajazimian S, Karamad V, Behroozi J, Sabet MN. Enhanced anticancer potency of hydroxytyrosol and curcumin by PLGA-PAA nano-encapsulation on PANC-1 pancreatic cancer cell line. Environ Toxicol. 2021; 36(6):1043-51.

18. Isazadeh A, Hajazimian S, Shadman B, Safaei S, Bedoustani AB, Chavoshi R, Shanehbandi D, Mashayekhi M, Nahaei M, Baradaran B. Anti-cancer Effects of Probiotic Lactobacillus acidophilus for Colorectal Cancer Cell Line Caco-2 through Apoptosis Induction. Pharmac Sci. 2020; 27(2):262-7. 
19. Huang CY, Ju DT, Chang CF, Reddy PM, Velmurugan BK. A review on the effects of current chemotherapy drugs and natural agents in treating non-small cell lung cancer. Biomedicine. 2017; 7(4).

20. Parikh NR, Mandal A, Bhatia D, Siveen KS, Sethi G, Bishayee A. Oleanane triterpenoids in the prevention and therapy of breast cancer: current evidence and future perspectives. Phytochemrev. 2014; 13(4):793-810.

21. Xu T, Pang Q, Wang Y, Yan X. Betulinic acid induces apoptosis by regulating PI3K/Akt signaling and mitochondrial pathways in human cervical cancer cells. Int j mol med. 2017; 40(6):1669-78.

22. Wang L, Wu J, Lu J, Ma R, Sun D, Tang J. Regulation of the cell cycle and PI3K/Akt/mTOR signaling pathway by tanshinone I in human breast cancer cell lines. Mol med rep. 2015; 11(2):931-9.

23. Feng $L$, Au-Yeung $W, X u Y H$, Wang SS, Zhu Q, Xiang P. Oleanolic acid from Prunella Vulgaris $L$. Induces SPC-A-1 cell line apoptosis via regulation of Bax, Bad and Bcl-2 Expression. Asian Pac J Cancer Prev. 2011; 12(2):403.

24. Fasano C, Disciglio V, Bertora S, Lepore Signorile M, Simone C. FOXO3a from the nucleus to the mitochondria: a round trip in cellular stress response. Cells. 2019; 8(9):1110.

25. Deeb D, Gao X, Jiang H, Dulchavsky SA, Gautam SC. Oleanane Triterpenoid CDDO-Me inhibits growth and induces apoptosis in prostate cancer cells by independently targeting pro-survival Akt and mTOR. Prostate. 2009; 69(8):851-60.

26. Liang J, Slingerland JM. Multiple roles of the PI3K/PKB (Akt) pathway in cell cycle progression. Cell cycle. 2003; 2(4):336-42.

27. Li X, Hu X, Wang J, Xu W, Yi C, Ma R, Jiang H. Inhibition of autophagy via activation of $\mathrm{PI} 3 \mathrm{~K} / \mathrm{Akt} / \mathrm{mTOR}$ pathway contributes to the protection of hesperidin against myocardial ischemia/reperfusion injury. Int J Mol Med. 2018; 42(4):1917-24.

28. Wang H, Liu Y, Wang D, Xu Y, Dong R, Yang Y, Lv Q, Chen X, Zhang Z. The upstream pathway of mTOR-mediated autophagy in liver diseases. Cells. 2019; 8(12):1597.

29. Wei Y, Liu M, Li X, Liu J, Li H. Origin of the autophagosome membrane in mammals. Biomed Res Int. 2018; 2018.

30. Liu J, Zheng L, Zhong J, Wu N, Liu G, Lin X. Oleanolic acid induces protective autophagy in cancer cells through the JNK and mTOR pathways. Oncol rep. 2014; 32(2):567-72.

31. Žiberna L, Šamec D, Mocan A, Nabavi SF, Bishayee A, Farooqi AA, Sureda A, Nabavi SM. Oleanolic acid alters multiple cell signaling pathways: implication in cancer prevention and therapy. Int $\mathrm{j}$ mol sci. 2017; 18(3):643.

32. Wang, T., Liu, C. and Jia, L. The roles of PKCs in regulating autophagy. J Cancer Res Clin Oncol, 144 (2018), pp.2303-2311.

\section{Figures}




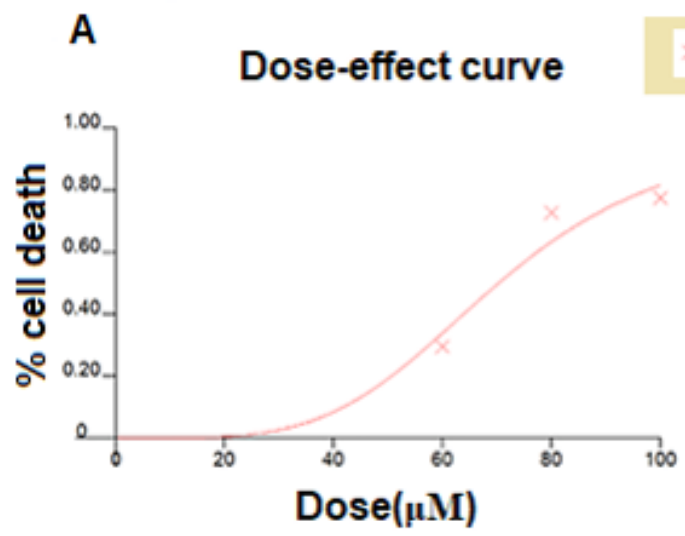

MA (72h)

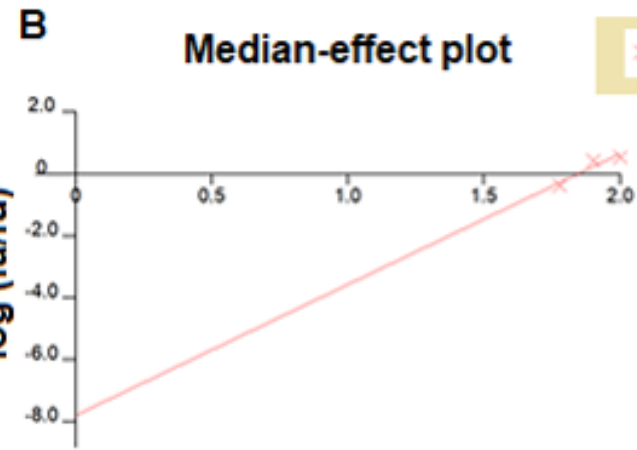

MA (72h)

$\log (D)$

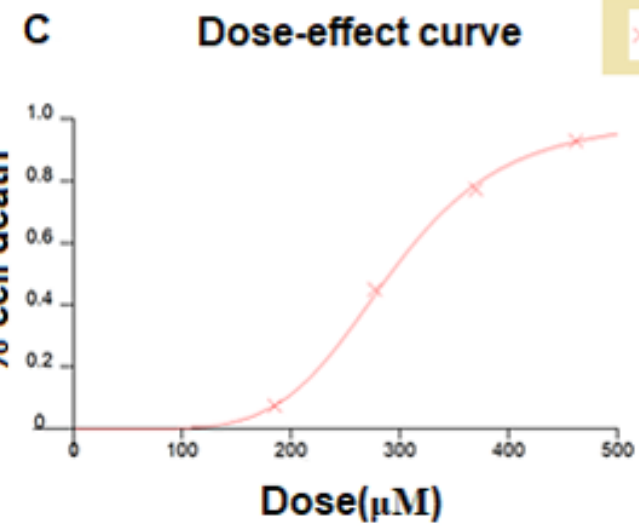

OA (72h)

D Median-effect plot

OA (72h)

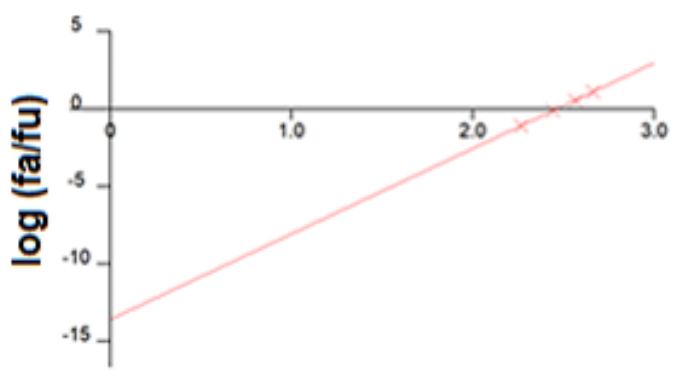

$\log (D)$

\section{Figure 1}

Dose effect curve, Median effect curve of MCF7 cell line for A, B) Maslinic acid (70.3 $\mu$ M) and C, D) Oleanolic acid $(291 \mu \mathrm{M})$.

A

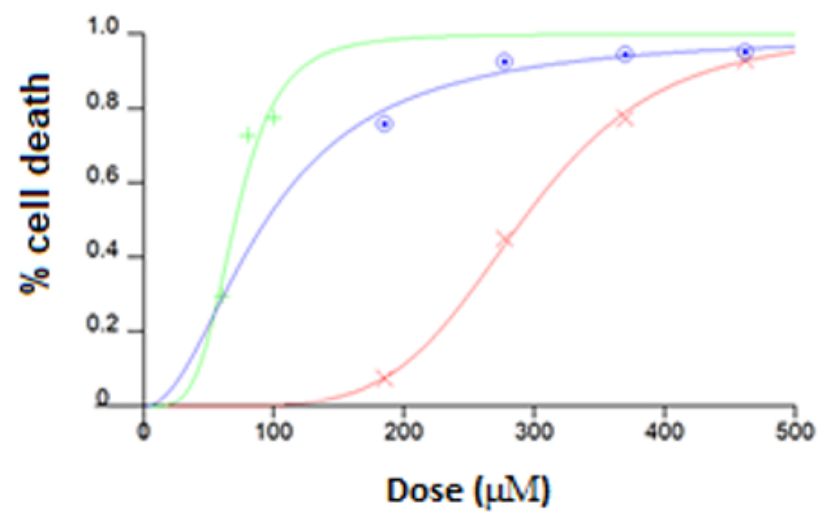

$O A(72 h) \times M A(72 h) \odot M A+O A(72 h)$
B Median-effect plot

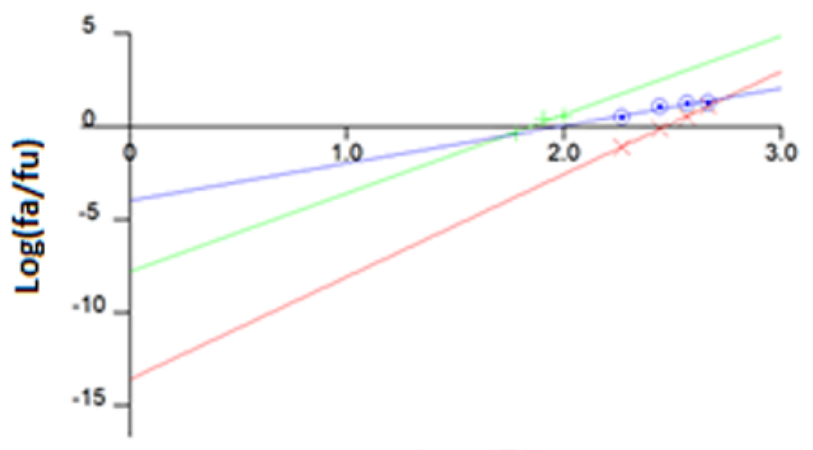

$\log (D)$

$O A(72 h) \times M A(72 h)-M A+O A(72 h)$ 


\section{Figure 2}

Dose effect curve, Median effect curve of MCF7 cell line for isobologram analysis for A, B) the combination of Maslinic acid $(20.34 \mu \mathrm{M})$ and Oleanolic acid $(94.01 \mu \mathrm{M})$.

\section{Figure 3}

Apoptosis analysis in flow cytometry, MCF7, Maslinic acid; Oleanolic acid; Combination. A) Control group,

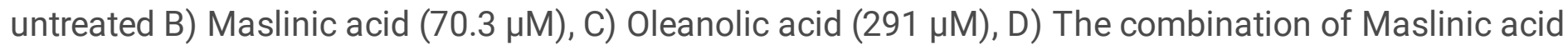
$(20.34 \mu \mathrm{M})$ and Oleanolic acid $(94.01 \mu \mathrm{M})$.

\section{Figure 4}

To measure the amount of autophagosome formation from GFP-labeled LC3B-expressing cells using a TR-FRET immunoassay approach. Determining autophagosome formation in the control group with maslinic acid, oleanolic acid and the combination group as a fold change. A) (The 1.74-fold increase) maslinic acid $(70.3 \mu \mathrm{M}), \mathrm{B})$ (the 0.37 -fold increase) oleanolic acid $(291 \mu \mathrm{M}), \mathrm{C})$ (the 3.25 -fold increase) the combination of maslinic acid $(20.34 \mu \mathrm{M})$ and oleanolic acid $(94.01 \mu \mathrm{M})$. ${ }^{\star \star} \mathrm{p}<0.001$ for all by oneway ANOVA

\section{Figure 5}

Cell cycle analysis in flow cytometry, MCF7, Maslinic acid; Oleanolic acid; Combination. A) Control group,

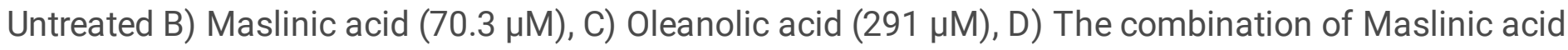
$(20.34 \mu \mathrm{M})$ and Oleanolic acid $(94.01 \mu \mathrm{M})$. 

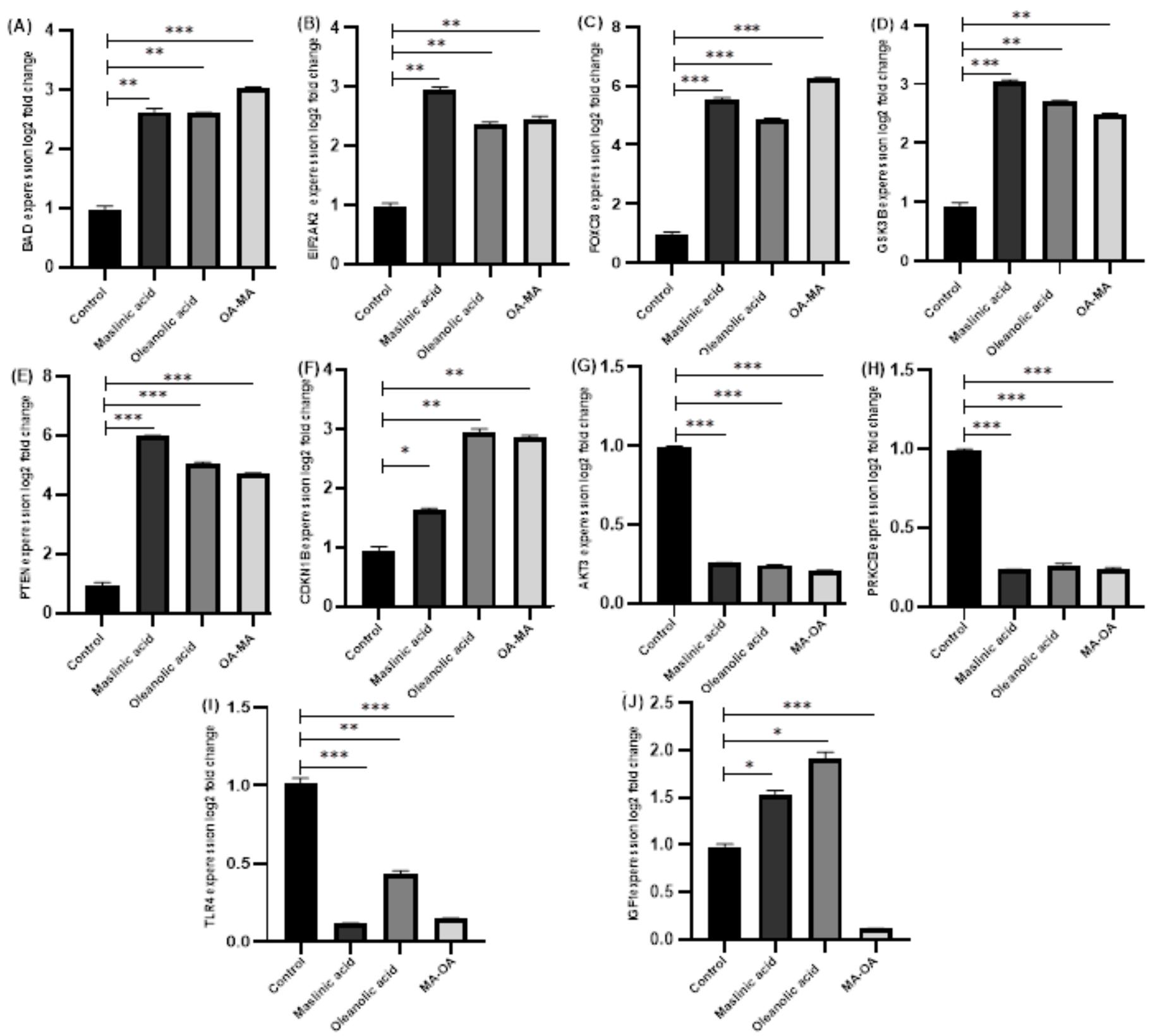

\section{Figure 6}

Changes in gene expression BAD (A), EIF2AK2 (B), FOXO3 (C), GSK3B (D), PTEN (E), CDKN1B (F), AKT3 $(\mathrm{G})$, PRKCB (H), TLR4 (I), and IGF1 (J) in MCF7 cell line treated with Maslinic acid, Oleanolic acid, and MAOA.

Figure 7

Schematic of the PI3K / AKT / mTOR pathway. Different proteins are involved in the PI3K / AKT / mTOR signal pathway so their genes can be affected by the appropriate drugs. 